\title{
Cavity longitudinal loss factor measurement by means of a beam test facility
}

\author{
A. Palmieri and L. Tecchio \\ INFN Laboratori Nazionali di Legnaro, via Romea 4, I-35020 Legnaro (PD), Italy \\ G. V. Lamanna and V. Variale \\ INFN Bari, Via E. Orabona 4, I-70126 Bari, Italy \\ A. V. Aleksandrov and P. V. Logatchov \\ Budker Institute of Nuclear Physics, 11 Lavrentiev Prospekt, Novosibirsk, 630090, Russian Federation \\ V. G. Vaccaro \\ Università degli Studi di Napoli "Federico II" and INFN Napoli, Via Cinthia 45 (Monte S. Angelo), I-80126 Napoli, Italy \\ M. R. Masullo \\ INFN Napoli, Via Cinthia 45 (Monte S. Angelo), I-81026 Napoli, Italy
}

(Received 20 June 2000; published 20 November 2000)

\begin{abstract}
A new method for the measurement of loss factor for an rf cavity is presented. The method consists of measuring the above quantity by means of the detection of both the rf voltage induced by an electron bunch in the device under test and the bunch charge. The device to be investigated is a copper reentrant T-shaped cavity. The experimental results and their comparison with analytical and numerical results are presented.

PACS numbers: 29.27.Bd
\end{abstract}

\section{INTRODUCTION}

Improvement of beam cooling techniques such as laser cooling allows the achievement of very cold ion beams inside storage rings. Moreover, with the appropriate cooling force, ordered ion structures, the so-called Coulomb crystals [1] can be obtained. One of the most important requirements that an ion ring devoted to such a purpose should fulfill is to avoid every kind of coherent instability that may cause beam losses [2]. One of these instabilities is related to beam-environment interaction by means of the longitudinal coupling impedance (LCI ) and the loss factor (LF) [3]. Therefore, a precise knowledge of such a quantity allows a more accurate estimation of instability growth rate and, in turn, the cooling rate needed.

Usually CI and LF measurements are performed in a laboratory using short current pulses propagating on a wire inside the accelerator element under test (coaxial wire method) [4], but this method is questionable for two reasons: (i) the electromagnetic properties of an empty chamber differs from a chamber with a wire inside and (ii) the coaxial wire method is not straightforward to use for velocities $\beta<1$, as in the case of cooled ion beams.

The main feature of our experiment is the indirect measurement of LF with an electron beam whose energy varies in the range $18-65 \mathrm{keV}(0.37 \leq \beta \leq 0.69)$. The device under test is an rf reentrant $\mathrm{T}$-shaped copper cavity.

In this article, we will compare experimental results with those coming from a theoretical formulation. In fact, an analytical method for the LF calculation has been developed for any particle velocity and for some relevant accelerator structures [5].

$1098-4402 / 00 / 3(11) / 112001(6) \$ 15.00$

\section{THE EXPERIMENTAL METHOD: A DESCRIPTION}

Let us consider a resonant rf cavity inserted on a vacuum chamber and excited by a charged particle beam, passing through the cavity, whose current is supposed to be frequency modulated. The energy lost by the beam due to the field induced by the beam itself can be described in terms of the LF $k$ :

$$
k=\frac{1}{\pi} \int_{0}^{+\infty} Z_{r}(\omega) d \omega
$$

with $Z_{r}$ the real part of the longitudinal coupling impedance (see the Appendix).

In the neighborhood of a cavity resonant frequency $\omega_{n}$, the interval of integration is reduced to a small region around the resonance, leading to the following formulation for $k$ (as shown in the Appendix):

$$
k_{l n}=\frac{\omega_{n} R_{n}}{2 Q_{n}},
$$

where $R_{n}$ is the cavity shunt resistance and $Q_{n}$ is the quality factor of the $n$th mode.

It can be shown (see the Appendix) that, for a bunch of charge $q$ and spectral density $|F(\omega)|$, the LF is related to the energy stored in the $n$th mode $W_{n}$ after the bunch passage by means of the relation

$$
W_{n}=q^{2} k_{l n}\left|F\left(\omega_{n}\right)\right|^{2} \text {. }
$$

For a Gaussian particle distribution we can write $\left|F\left(\omega_{n}\right)\right|=\exp \left(-\omega_{n}^{2} \sigma^{2} / 2\right)$ where $\sigma$ is the rms temporal bunch width.

(C) 2000 The American Physical Society

$112001-1$ 
Let us now consider an external measurement line connected to the cavity. The energy balance for the $n$th mode gives us the following relation, valid for a mode slowly decaying with respect to the beam transit time:

$$
\frac{d W_{n}}{d t}=-\frac{W_{n}}{\tau_{n}}=-\left(P_{l n}+P_{\text {ext }}\right)
$$

where $\tau_{n}=\frac{Q_{L n}}{\omega_{n}}$ is the decay time of the $n$th mode, $P_{\text {in }}$ is the power dissipated inside the cavity, $P_{\text {ext }}$ is the power radiated in the measurement line. $Q_{L n}$ is the "loaded quality factor" which takes into account the power flowing towards the measurement line; it turns out to be

$$
Q_{L n}=\omega_{n} \frac{W_{n}}{P_{\mathrm{in}}+P_{\mathrm{ext}}} .
$$

The peak voltage $U_{\text {rf }}$ induced in the measurement line with impedance $R$ is $U_{\mathrm{rf}}=\sqrt{2 R P_{\mathrm{ext}}}$. Using Eqs. (3) and (4) we get

$$
U_{\mathrm{rf}}(q)=\sqrt{\frac{2 R k_{\ln } \alpha_{n}\left|F\left(\omega_{n}\right)\right|^{2}}{\left(1+\alpha_{n}\right) \tau_{n}}} q=r_{n} q,
$$

where we have introduced the coupling coefficient $\alpha_{n}$ defined as $\alpha_{n}=\frac{P_{\text {ext }}}{P_{\text {in }}}$

Equation (5) shows a linear dependence between the rf voltage and the beam charge. It is very important to point out that this linear relationship holds as far as the time bunch length stays constant. If that is not the case, Eq. (5) must be modified in order to take into account bunch lengthening due to space charge forces and laser instability. If we assume that space charge effects are a first-order correction with respect to the "unperturbed" bunch duration $\sigma_{0}$, we obtain

$$
\sigma=\sigma_{0}+a q,
$$

where the angular coefficient $a$ takes into account the way in which bunch duration is modified by the space charge.

Therefore, by substituting into Eq. (5), we get

$$
\begin{aligned}
U_{\mathrm{rf}}(q)= & \sqrt{\frac{2 k_{\ln } \alpha_{n} \exp \left(-\omega_{n}^{2} \sigma_{0}^{2}\right) R}{\left(1+\alpha_{n}\right) \tau_{n}}} \\
& \times q \exp \left[-\frac{\omega_{n}^{2}}{2} a q\left(2 \sigma_{0}+a q\right)\right] \\
= & r_{n} q \exp \left[-\frac{\omega_{n}^{2}}{2} a q\left(2 \sigma_{0}+a q\right)\right] .
\end{aligned}
$$

This equation tells us that the dependence of the induced rf voltage on the charge $q$ can be described by means of two parts: the first one, linear, containing in the coefficient $r_{n}$ the loss factor and so the "interaction" beam cavity; a second one, exponential, due to the effect of space charge on the bunch length and on the time spent in the cavity.

The LF can be extracted from $r_{n}$ as follows:

$$
k_{l n}=r_{n}^{2} \frac{\left(1+\alpha_{n}\right) \tau_{n}}{2 R \alpha_{n}\left|F\left(\omega_{n}\right)\right|^{2}} .
$$

The relations (7) and (8) give us the base for the setting up of the experimental measurement method.

The induced rf voltage in the cavity can be measured as a function of the incoming beam charge by varying its value. At the same time and separately the beam charge has to be measured. In this way, an experimental relation between the two quantities can then be found. By means of Eq. (7), the data $\left(q, U_{\mathrm{rf}}\right)$ are interpolated varying the two parameters $r_{n}$ and $\alpha_{n}$; the LF can then be calculated from the coefficient $r_{n}$ [see Eq. (8)] once $\alpha_{n}$ and $\tau_{n}$ have been measured. Therefore, we get the loss factor for a given resonant mode frequency and for a fixed beam energy.

Changing the beam energy, the couple of data $\left(q, U_{\mathrm{rf}}\right)$ is measured again as before and a new value of LF can be found. The same has to be done to study the behavior of $k_{n}$ as a function of the frequency.

\section{EXPERIMENTAL APPARATUS AND TECHNIQUE}

From the above discussion it is clear that, as far as Eq. (7) holds, by measuring several times, independently, the induced rf voltage in the cavity and the amount of beam charge passing through the cavity, it is possible to interpolate the data and to extract the required LF from the coefficient $r_{n}$.

The experimental setup is shown in Fig. 1.

A bunched electron beam is emitted by a GaAs photocathode excited by a frequency doubled Nd:YLF laser. The measured rms pulse duration of photon bunch is $\sigma_{0 \text { photon }}=(70 \pm 10) \mathrm{ps}$. The photocathode is installed in a Pierce type electron gun. A voltage applied between anode and cathode accelerates the bunch. By varying the anode-cathode voltage it is possible to perform measurements for different values of the particle energy and therefore of the velocity, $\beta$.

A Faraday cup (FC), put at the end of the measurement line, is used to collect and measure the bunch charge $q$ passing through the cavity.

The beam transport to the device under test (DUT) (rf cavity in our case) and then to the FC is accomplished by using a magnetic lens system.

By varying the laser intensity by means of polaroid filters the photoemitted current changes; in correspondence of this, beam charge intensity varies from (1.2 \pm $0.06) \times 10^{7}$ electrons (minimum photoemitted charge) to $(4.2 \pm 0.21) \times 10^{8}$ electrons (maximum photoemitted charge). Following our assumption [Eq. (6)], the rms "unperturbed" electron bunch duration $\sigma_{0}$ is equal to $\sigma_{0 \text { photon }}$.

Since the proposed experimental technique is valid only around cavity resonances, as a first step we must measure the cavity resonance frequencies and relative loaded quality factors without beam flowing. For our experiment we chose two TM resonant frequencies whose measured 


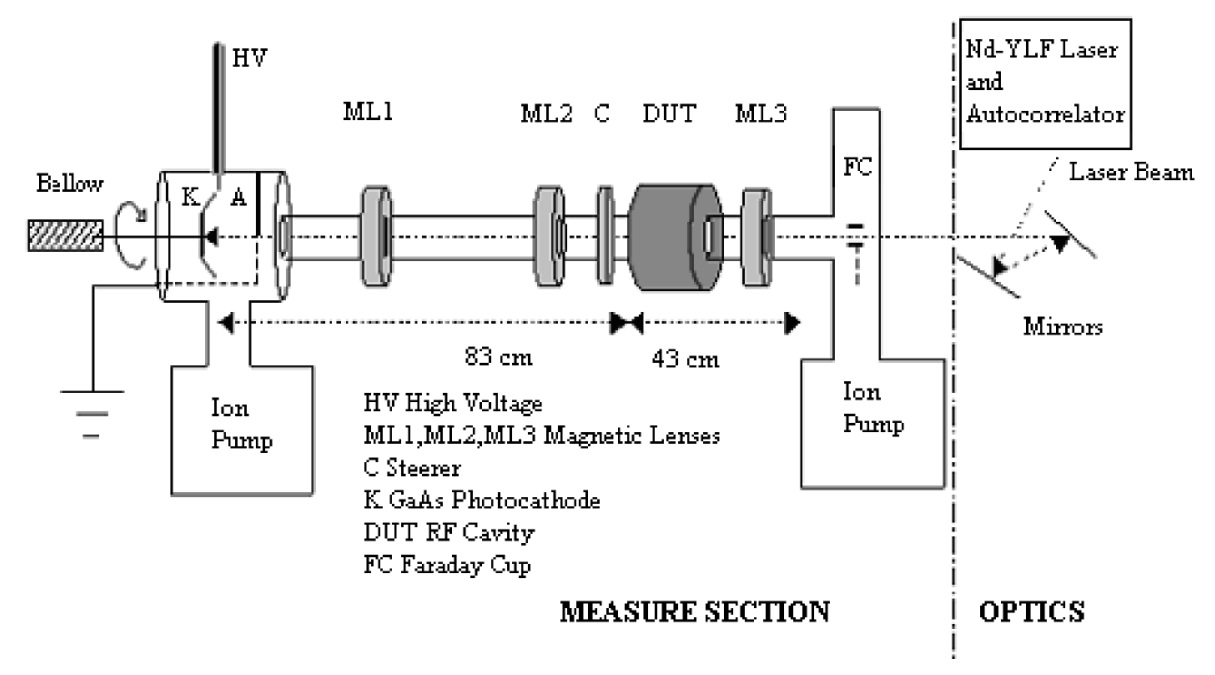

FIG. 1. The experimental setup for the longitudinal loss factor (LLF) measurement.

values, corresponding loaded quality factors, and relative decay time are shown in Table I.

The induced rf voltage in the cavity and the charge collected on the FC are measured with two separated lines at the same time, as shown in Fig. 2. For this purpose, high voltage (HV) and laser triggers are synchronized by means of a pulse generator with a repetition rate of $0.5 \mathrm{~Hz}$.

In order to properly reconstruct the rf signal from the cavity, we need to acquire its entire frequency band. Looking at Table I it is clear that the filters usually installed inside the spectrum analyzer (maximum bandwidth of $3 \mathrm{MHz}$ ) are not sufficient. For this reason we had to properly customize a second intermediate frequency (IF) output on our spectrum by inserting a $30 \mathrm{MHz}$ bandwidth filter. In this way we were able to acquire the entire rf signal inside the cavity at the working frequencies. The output signal amplified by an rf amplifier is then read out on a LeCroy oscilloscope.

The output signal $u_{\mathrm{rf}}^{\prime}(t)$, measured on the oscilloscope, is

$$
u_{\mathrm{rf}}^{\prime}(t)=U_{\mathrm{rf}} \exp \left(-\Gamma_{n}^{\prime} t\right)\left[\cos \left(\bar{\omega}_{n}^{\prime} t\right)-\frac{\Gamma_{n}^{\prime}}{\bar{\omega}_{n}^{\prime}} \sin \left(\bar{\omega}_{n}^{\prime} t\right)\right],
$$

with $\bar{\omega}_{n}^{\prime}=2 \pi f_{0}\left(1-\frac{1}{4 Q_{n}^{2}}\right) \quad$ and $\quad \Gamma_{n}^{\prime}=\frac{\pi f_{0}}{Q_{n}}$.

From this equation, $U_{\mathrm{rf}}$ can be obtained and introduced in Eq. (7).

TABLE I. Loaded $Q$ 's, coupling factors, and decay times for the two resonant frequencies.

\begin{tabular}{llrcc}
\hline \hline$n$ & $f_{n}(\mathrm{GHz})$ & $Q_{L n}$ & $\alpha_{n}$ & $\tau_{n}(\mathrm{~ns})$ \\
\hline 1 & 0.8567 & 1178 & 0.2 & 438 \\
2 & 2.361 & 595 & 0.5 & 80 \\
\hline \hline
\end{tabular}

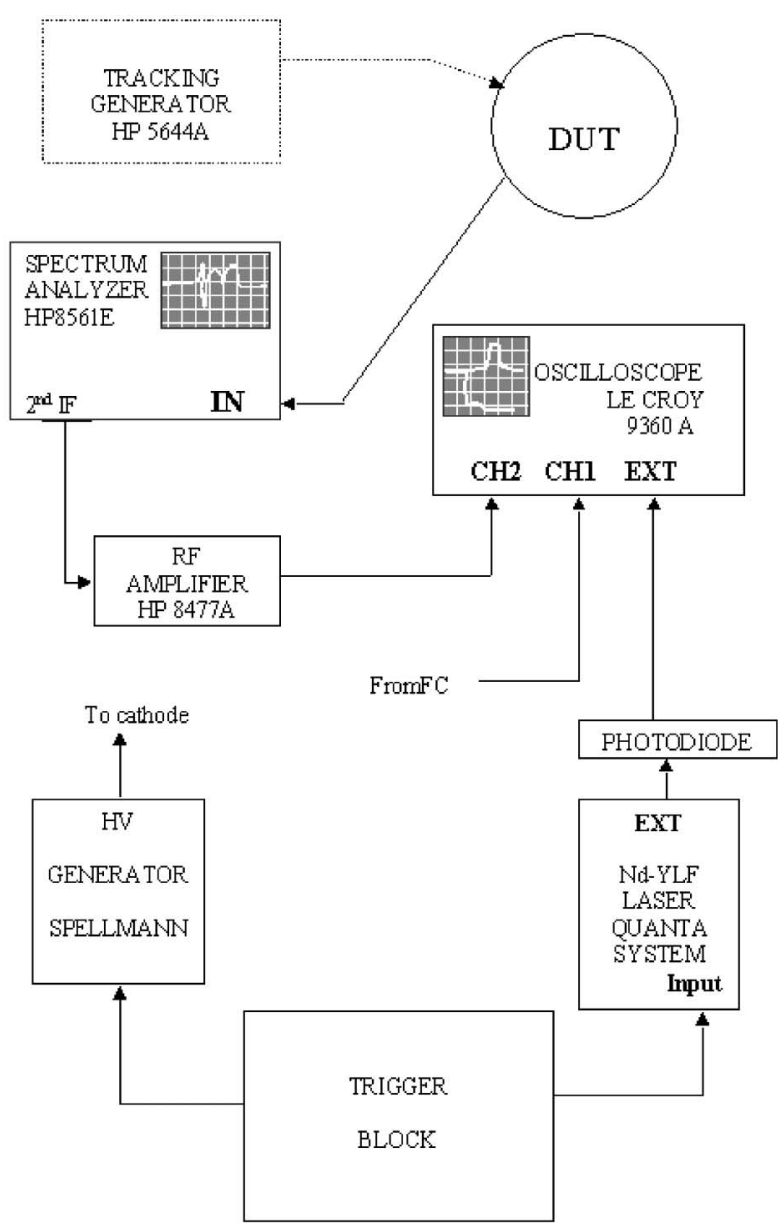

FIG. 2. Scheme for the simultaneous determination of $U_{\text {rf }}$ and $U_{\mathrm{FC}}$. 


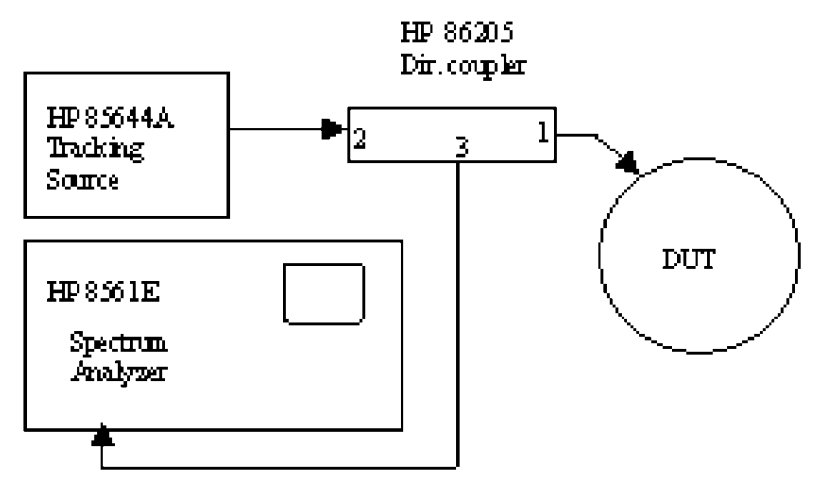

FIG. 3. Scheme for the reflection coefficient measurement.

The charge $q$ can be calculated by measuring the voltage $U_{\mathrm{FC}}$ induced on $\mathrm{FC}$ by the electron bunch through the relation $q=C_{\mathrm{FC}} U_{\mathrm{FC}}$, where $C_{\mathrm{FC}}=667 \mathrm{pF}$ is the capacitance of the Faraday cup.

The coupling $\alpha_{n}$ appearing in Eq. (7) can be determined from the measurement of the reflection coefficient $\rho_{n}$ through the relation [6]

$$
\alpha_{n}= \begin{cases}\frac{1-\left|\rho_{n}\right|}{1+\left|\rho_{n}\right|}, & \text { if } \alpha_{n}<1 \\ \frac{1+\left|\rho_{n}\right|}{1-\left|\rho_{n}\right|}, & \text { if } \alpha_{n} \geq 1\end{cases}
$$

For both working frequencies, the reflection coefficients have been measured according to the scheme shown in Fig. 3. $\alpha_{n}$ values are reported in Table I.

As the final step, we need to know the frequency response of the $U_{\text {rf }}$ measurement line without beam flowing according to the scheme shown in Fig. 4, in order to take into account cable attenuation and amplification.

The frequency response is given by

$$
H(f)=\frac{V_{\text {out }}(f)}{V_{\text {in }}(f)} .
$$

For each resonant frequency, the "true" value of $r_{n}$ is related to the measured value by means of the relation $r_{n}^{\text {true }}=r_{n}^{\text {measured }} / H\left(f_{n}\right)$.
TABLE II. Response of the $q-U_{\mathrm{rf}}$ measurement line at the operating frequencies.

\begin{tabular}{lll}
\hline \hline$n$ & $f_{n}(\mathrm{GHz})$ & $H\left(f_{n}\right)$ \\
\hline 1 & 0.8567 & 2.068 \\
2 & 2.361 & 1.312 \\
\hline \hline
\end{tabular}

The measured values of $H\left(f_{n}\right)$ are shown in Table II.

\section{EXPERIMENTAL RESULTS AND COMPARISON WITH THEORY}

We have measured the values of $q$ and $U_{\mathrm{rf}}(q)$ for different values of beam energies and for two resonant frequencies. For each of the beam energies, the couples $\left[q-U_{\mathrm{rf}}(q)\right]$ have been interpolated according to Eq. (7).

We can write Eq. (7) as

$$
y(x)=x \exp \left(\alpha x^{2}+\beta x+\gamma\right),
$$

with $x=q, y=U, \alpha=-\frac{\omega_{n}^{2} a^{2}}{2}, \beta=-\omega_{n}^{2} a \sigma_{0}, \gamma=$ $\ln \left(r_{n}\right)$.

Provided $x>0$ and $y>0$, we can linearize Eq. (11) with respect to the fitting parameters obtaining

$$
Y=\ln (y / x)=\alpha x^{2}+\beta x+\gamma .
$$

Therefore, by fitting with the least squares method the couples $(Y, x)$ according to Eq. (12), we can obtain the best value for $\gamma$ and then for $r_{n}$.

A comparison between experimental and theoretical data for each measured frequency is shown in Figs. 5 and 6 as a function of beam energy. The first theoretical curve is the result of a simulation performed by using a modified version of the URMEL code. The second is the result of the calculation of LF for our T-shaped lossy cavity with a new formulation of mode matching technique [7]. The error bars, calculated by means of error propagation, are of $15 \%$ for the first frequency and $14 \%$ for the second one. In the evaluation of these errors we have considered the dependence of $k_{l n}$ on $\alpha_{n}, \tau_{n}, r_{n}$, and $F\left(\omega_{n}\right)$, as shown in Eq. (8).

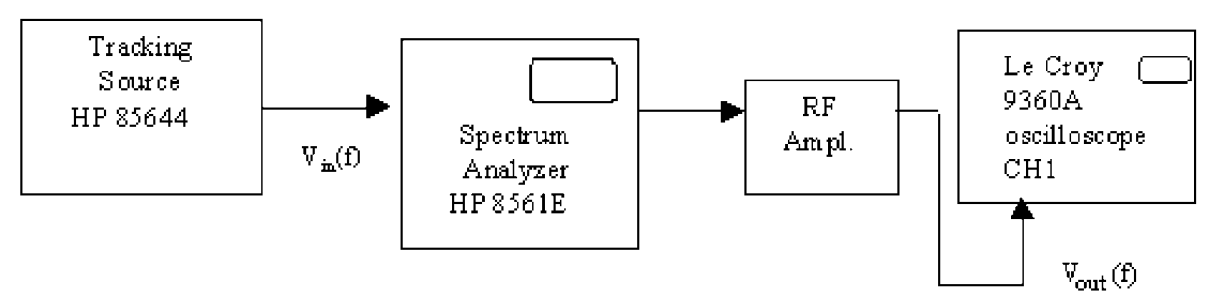

FIG. 4. Scheme of the frequency response measurement line. 


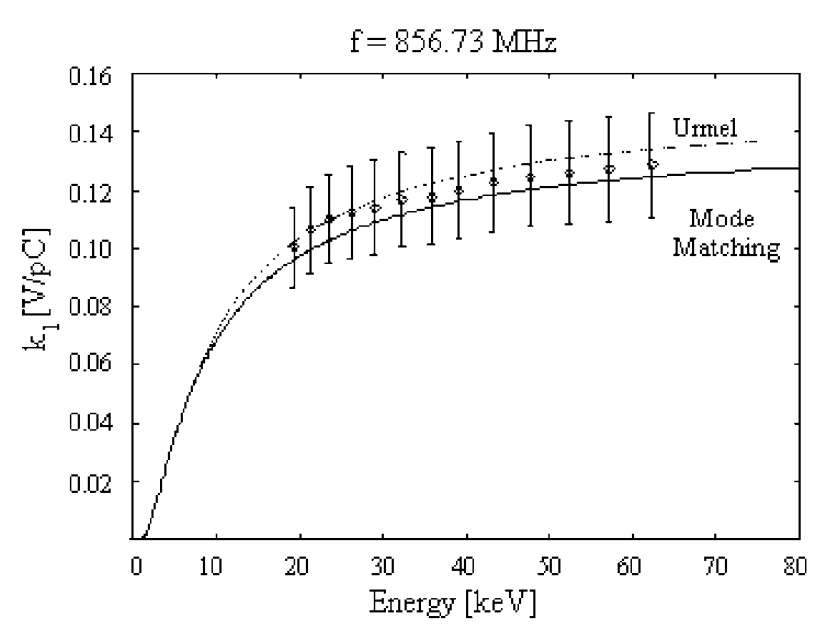

FIG. 5. Loss factor as a function of bunch energy; comparison between theoretical and experimental results at $f=856.73 \mathrm{MHz}$.

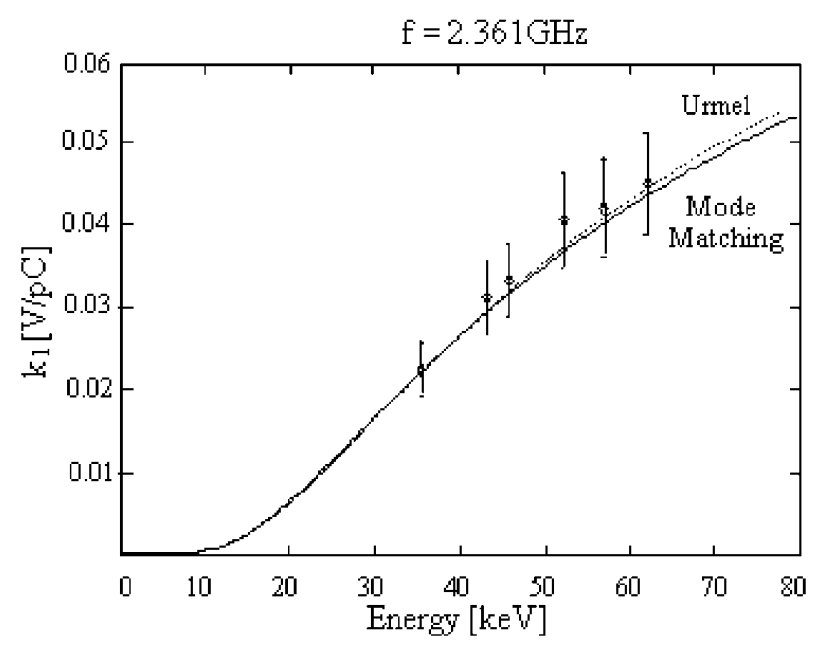

FIG. 6. Loss factor as a function of bunch energy; comparison between theoretical and experimental results at $f=2361 \mathrm{MHz}$.

\section{CONCLUSIONS AND PERSPECTIVES}

The experimental results and the theoretical evaluations turned out to be in good agreement. As a consequence, the proposed measurement method is reliable and useful for possible future applications. In particular, under certain circumstances it has been confirmed that nonrelativistic beams are favored in the case of coherent instabilities due to beam-environment interaction. At the same time we have to point out that further improvements are possible, especially with regard to measurement implementation. In particular, error bars can be reduced, in order to obtain more precise data. This can be achieved by using shorter and more stable laser pulses. In fact, for a fixed frequency and beam energy, in the case of laser intensity stability, the effect of space charge forces can be easily recognized [8]. Indeed, laser pulse duration stability reduces the error in the measurement of $F\left(\omega_{n}\right)$. Moreover, the shorter the laser pulse duration, the wider the region in which a linear relation between $U_{\mathrm{rf}}$ and $q$ is fulfilled. Consequently, the estimate of $r_{n}$ will be more accurate.

\section{APPENDIX: THE LONGITUDINAL LOSS FACTOR}

The dynamics of a particle beam traveling inside an accelerator is affected by the electromagnetic (e.m.) fields induced (wakefields) by the beam itself in the interaction with the vacuum chamber. This interaction can be described by means of the wake functions (potentials) in time domain or the longitudinal coupling impedance in frequency domain.

For a charged particle $q_{1}$, traveling with constant velocity $v(v=\beta c)$ along the axis $z$ of an arbitrary shape vacuum chamber, the electromagnetic energy lost is given by

$$
U\left(\mathbf{r}_{1}\right)=-\int_{-\infty}^{+\infty} \mathbf{F}\left(\mathbf{r}_{1}, z_{1}, t\right) \hat{\mathbf{z}} d z
$$

with $t=z_{1} / v$ and $\mathbf{F}$ the Lorentz force due the e.m. fields induced by the charge in the presence of discontinuities. The quantity $U$ takes into account both the energy lost in the resistive walls and the energy from the diffracted fields.

Let us define the LLF [9] as the energy lost by the charge $q_{1}$ per unit charge squared,

$$
k\left(\mathbf{r}_{1}\right)=\frac{U\left(\mathbf{r}_{1}\right)}{q_{1}^{2}} .
$$

Consider now a second particle $q$, displaced apart from $q_{1}$; its energy will change as a consequence of the interaction with the e.m. fields produced by the first particle by an amount

$$
U_{21}\left(\mathbf{r}_{1}, \mathbf{r}, \tau\right)=-\int_{-\infty}^{+\infty} \mathbf{F}\left(\mathbf{r}_{1}, \mathbf{r}, z_{1}, z, t\right) \hat{\mathbf{z}} d z
$$

where $\tau=t-z_{1} / v$ is the time delay between the two particles.

We define the longitudinal wake function as follows:

$$
w\left(\mathbf{r}, \mathbf{r}_{1}, \tau\right)=\frac{U_{21}\left(\mathbf{r}, \mathbf{r}_{1}, \tau\right)}{q_{1} q} .
$$

Let us notice that if $\beta<1$ and in the limit of zero distance between the two particles, then

$$
k=w_{z}(0) \text {. }
$$

In the frequency domain let us define the longitudinal coupling impedance as the Fourier transform of the longitudinal wake function

$$
Z\left(r_{1}, r, \omega\right)=\int_{-\infty}^{+\infty} w_{z}\left(r_{1}, r, \tau\right) e^{-j \omega \tau} d \tau .
$$

For simplicity, we will consider only the case $\mathbf{r}_{1}=\mathbf{r}=0$, thus omitting the radial dependence.

It is possible to relate $k$ and $Z(\omega)$ in the following way: 


$$
k=\frac{1}{\pi} \int_{0}^{+\infty} Z_{r}(\omega) d \omega
$$

Let us consider now a resonant cavity. In the neighborhood of a resonance frequency $\omega_{n}$, the cavity behaves like a $R L C$ parallel circuit for the particles; in this frame the longitudinal coupling impedance can be written as [10]

$$
Z(\omega)=\frac{R_{n}}{1+j Q_{n}\left(\frac{\omega}{\omega_{n}}-\frac{\omega_{n}}{\omega}\right)},
$$

where $R_{n}$ and $Q_{n}$ are the cavity shunt resistance and the unloaded quality factor of the $n$th resonant mode. $R_{n}$ is defined (see circuit theory) as the ratio of the accelerating voltage on the cavity axes to the power loss in the cavity walls.

If we are very close to the resonance $\omega_{n}$, by combining Eqs. (7) and (8), it is possible to write the longitudinal LF for the $n$th mode as

$$
k_{n}=\frac{\omega_{n} R_{n}}{2 Q_{n}}
$$

Consider now, instead of a point charge exciting the cavity, a distribution of particles $i(t)$ such that

$$
q_{1}=\int_{-\infty}^{+\infty} i(\tau) d \tau
$$

For this distribution, the longitudinal wake function $W(\tau)$ is simply the convolution product of $w(\tau)$ and $i(\tau)$,

$$
W(\mathbf{r}, \tau)=\frac{1}{q_{1}} i(\tau) * w(\mathbf{r}, \tau) .
$$

As a consequence, the bunch loss factor $K$ of this distribution is given by

$$
K(\mathbf{r})=\frac{1}{q_{1}} \int_{-\infty}^{+\infty} W(\mathbf{r}, \tau) i(\tau) d \tau
$$

In analogy with Eq. (2), we can express the energy lost by a charge distribution $i(\tau)$ as

$$
U=q_{1}^{2} K .
$$

Therefore, recalling Eq. (12) and the definition of LCI we can write

$$
U=\frac{1}{\pi} \int_{0}^{+\infty} Z_{R}(\omega)|I(\omega)|^{2} d \omega
$$

Let us assume now a bunch with a spectral distribution $I(\omega)=q F(\omega)$ which is nearly constant around the resonance frequency $\omega_{n}$. In this case, the e.m. energy lost in the $n$th mode can be expressed as a function of the loss factor, remembering Eq. (9),

$$
U_{n}=q_{1}^{2} k_{n}\left|F\left(\omega_{n}\right)\right|^{2} .
$$

[1] J. P. Schiffer, GSI Report No. 89-10, 1989.

[2] S. Cocher and I. Hoffman, Part. Accel. 34, 189 (1990).

[3] L. Palumbo, V. G. Vaccaro, and A. Zobov, in Proceedings of the CERN Accelerator School, Fifth Advanced Accelerator Physics Course, 1993, Rhodes, Greece, edited by S. Turner (CERN, Geneva, 1995), p. 332.

[4] M. Sands and A. Rees, SLAC Report No. PEP-0095, 1974.

[5] D. Davino, M. R. Masullo, A. Palmieri, and V. G. Vaccaro (to be published).

[6] T. P. Wangler, RF Linear Accelerators (Wiley, New York, 1998),Vol. I, Chap. 5, p. 139.

[7] Antonio Palmieri, thesis, Università degli Studi di Napoli "Federico II," 1998.

[8] A. V. Aleksandrov, R. Calabrese, G. Ciullo, N. S. Dikansky, V. Guidi, G. Lamanna, P. Lenisa, P. V. Logatchov, A. V. Novokhatsky, L. Tecchio, and B. Yang, Rev. Sci. Instrum. 66, 3363 (1995).

[9] L. Palumbo and V. G. Vaccaro, in Proceedings of the CERN Accelerator School, Advanced Accelerator Physics, 1986, Oxford, England, edited by S. Turner (CERN, Geneva, 1987), p. 360.

[10] G. Dôme, in RF Engineering for Particle Accelerators, 1991, edited by S. Turner (CERN, Geneva, 1992), p. 100. 